\title{
Reprodução de Trichospilus diatraeae Cherian \& Margabandhu (Hymenoptera: Eulophidae) em Pupas de Anticarsia gemmatalis Hübner (Lepidoptera: Noctuidae) em Relação à Idade do Parasitoide e Hospedeiro
}

Patrik Luiz Pastori'1, José Cola Zanuncio², Robson Oliveira Silva², Fabricio Fagundes Pereira ${ }^{3}$, Rosalia Azambuja ${ }^{\circledR}$ \& José Milton Milagres Pereira²

1. Universidade Federal do Ceará, e-mail: plpastori@yahoo.com.br 2. Universidade Federal de Viçosa, e-mail: jczanuncio@ufv.br, robson agro@yahoo.com.br, jmmpereir@ufv.br. 3. Universidade Federal da Grande Dourados, e-mail: fabriciofagundes@ufgd.edu.br, july_azambuja@yahoo.com.br (Autor para correspondência ${ }^{\varpi}$ ).

EntomoBrasilis 5 (1): 37-42 (2012)

Resumo. A reprodução de parasitoides apresenta etapas interligadas e a idade de hospedeiros e parasitoides pode afetar o desenvolvimento desses últimos. Características biológicas de fêmeas de Trichospilus diatraeae Cherian \& Margabandhu (Hymenoptera: Eulophidae), com até 96 horas de idade, foram estudadas em pupas do hospedeiro alternativo Anticarsia gemmatalis Hübner (Lepidoptera: Noctuidae). Cada pupa de A. gemmatalis com 0-24, 24-48, 48-72 ou 72-96 horas de idade, foi oferecida ao parasitismo por 10 fêmeas de $T$. diatraeae com 0-24, 24-48, 48-72 ou 72-96 horas de idade, por 24 horas à $25 \pm 2^{\circ} \mathrm{C}$, umidade relativa de $70 \pm 10 \%$ e fotofase de 14 horas. A emergência, o número de descendentes, a duração do ciclo, a razão sexual, a longevidade e largura da cápsula cefálica de machos e de fêmeas de $T$. diatraeae apresentaram resposta semelhante para o efeito idade das pupas desse hospedeiro e fêmeas desse parasitoide em conjunto. No entanto, houve redução da emergência, número de descendentes e longevidade das fêmeas de $T$. diatraeae com duração do ciclo se ajustando a uma função quadrática quando se avaliou apenas o efeito da idade do parasitoide. Pupas de A. gemmatalis, com até 96 horas de idade, podem ser usadas para criação massal de T. diatraeae utilizando-se fêmeas desse parasitoide com até 96 horas de idade.

Palavras-chave: Criação massal; hospedeiro alternativo; resposta funcional.

\section{Reproduction of Trichospilus diatraeae Cherian \& Margabandhu (Hymenoptera: Eulophidae) on Anticarsia gemmatalis Hübner (Lepidoptera: Noctuidae) Pupae in Relation to Age of the Parasitoid and Host}

Abstract. Reproduction of parasitoids presents interconnected steps and the ages of hosts and parasitoids can affect the development of parasitoids. Biological parameters of Trichospilus diatraeae Cherian \& Margabandhu (Hymenoptera: Eulophidae), at up to 96 hours of age were studied on pupae of the alternative host Anticarsia gemmatalis Hübner (Lepidoptera: Noctuidae) pupae. Each A. gemmatalis pupae from 0-24, 24-48, 48-72 or 72-96 hours of age, was offered to parasitism to 10 females of $T$. diatraeae at 0-24, 24-48, 48-72 or 72-96 hours of age for 24 hours at $25 \pm 2^{\circ} \mathrm{C}$, relative humidity of $70 \pm 10 \%$ and photophase of 14 hours. Emergency, number of offspring, length of cycle, sex ratio, longevity and width of cephalic capsule of males and females of $T$. diatraeae showed similar response when effect of age of pupae and females of parasitoid were evaluate jointly. However, emergency, number of offspring and longevity of females of $T$. diatraeae were reduced as length of cycle was being adjusted to a quadratic function when only effect of age of parasitoid was evaluated. Pupae of A. gemmatalis with up to 96 hours of age can be used for mass rearing of $T$. diatraeae using females at up to 96 hours of age.

Keywords: Alternative host; functional response; mass rearing.

$\mathbf{F}$ êmeas de parasitoides de vida livre possuem mecanismos para escolha de hospedeiros adequados para suas fases imaturas (IMANDEH 2006; NofEMela \& KFIR 2008). A oviposição no hospedeiro é determinada por mecanismos olfativos, visuais, táteis, gustativos, sinais químicos, tamanho, idade e estado nutricional do hospedeiro (HARVEY \& STRAND 2002; NOFEMELA \& KFIR 2008) e as fêmeas parasitam o maior hospedeiro disponível para proporcionar mais recursos a seus descendentes (LIN \& Ives 2003).

O parasitismo apresenta etapas interligadas (WANG \& LiU 2002) e a experiência e idade do parasitoide podem influenciar o comportamento de oviposição (CoOPERBAND et al. 2003; CHONG \& Oetring 2007). Parasitoides freqüentemente parasitam o hospedeiro na fase/idade mais favorável a sobrevivência dos imaturos visando o desenvolvimento rápido e produção de adultos maiores (HARveY \& STRAND 2002; WANG \& LiU 2002). Contudo, a qualidade nutricional de pupas hospedeiras pode diminuir com a idade (WANG \& LIU 2002).

As fêmeas de Trichospilus diatraeae Cherian \& Margabandhu (Hymenoptera: Eulophidae) utilizam hospedeiros de várias ordens de insetos incluindo pupas de Lepidoptera (PARON \& BERTI Filho 2000; PEReira et al. 2008; ZaChé et al. 2010). T. diatraeae é agente potencial no controle biológico de pragas de cana-deaçúcar, milho e algodão em países da África, Âsia e Américas (BoUČEK 1976; FÁvero 2009; GranCE 2010; Rodrigues 2010).

O sucesso de programas de controle biológico com parasitoides depende de interações comportamentais e fisiológicas entre parasitoides e hospedeiros (AMALIN et al. 2005; PASTORI et al. 2010) e isto pode ser melhorado pelo conhecimento de características 
físicas, químicas e biológicas, incluindo fecundidade, razão sexual e ecologia do hospedeiro e do parasitoide (UÇKAN \& GÜLEL 2002; AMALIN et al. 2005).

A reprodução de fêmeas de $T$. diatraeae foi avaliada em pupas do hospedeiro alternativo Anticarsia gemmatalis Hübner (Lepidoptera: Noctuidae) considerando as idades do parasitoide e hospedeiro visando aprimorar o procedimento de criação massal desse parasitoide.

\section{MATERIAL E MÉTODOS}

O experimento foi conduzido no Laboratório de Controle Biológico de Insetos (LCBI) do Instituto de Biotecnologia Aplicada a Agricultura (BIOAGRO) da Universidade Federal de Viçosa (UFV) em Viçosa, Minas Gerais, com as etapas:

Criação de A. gemmatalis. Lagartas de A. gemmatalis foram criadas em potes plásticos $(1.000 \mathrm{~mL})$ com a tampa furada e vedada com organza e alimentadas com dieta artificial proposta por GreEne et al. (1976) à $25 \pm 2^{\circ} \mathrm{C}, 70 \pm 10 \%$ de umidade relativa e fotofase de 14 horas. Os adultos foram mantidos em gaiolas de madeira ( $33 \times 33 \times 33 \mathrm{~cm}$ ) com as laterais teladas e fechadas com tampa de vidro. Esses adultos foram alimentados com solução nutritiva embebida em algodão (GREENE et al. 1976). As posturas foram coletadas em folhas de papel branco dispostas no interior das gaiolas, recortadas e colocadas nos potes com a dieta artificial para continuidade da criação em laboratório.

Criação de $T$. diatraeae. Os adultos de $T$. diatraeae da criação do LCBI foram mantidos em tubos de vidro (14,0 x 2,2 $\mathrm{cm}$ ), fechados com algodão e alimentados com gotículas de mel puro. Uma pupa de A. gemmatalis foi oferecida as fêmeas de $T$. diatraeae recém-emergidas por 24 horas e, após esse período, individualizadas e mantidas a $25 \pm 2^{\circ} \mathrm{C}, 70 \pm 10 \%$ de umidade relativa (RODRIGUES 2010) e fotofase de 14 horas até a emergência de seus adultos (PEREIRA et al. 2008).

Desenvolvimento experimental. Cada pupa de $A$. gemmatalis com 0-24, 24-48, 48-72 ou 72-96 horas de idade foi pesada (202,33 $\pm 11,37 \mathrm{mg})$ e exposta ao parasitismo por 10 fêmeas de $T$. diatraeae com 0-24, 24-48, 48-72 ou 72-96 horas de idade. As fêmeas do parasitoide foram alimentadas com mel puro e o número utilizado foi determinado em teste preliminar em função do peso das pupas. Parasitoides e hospedeiros foram acondicionados em tubos de vidro (14,0 x 2,2 cm) tampados com algodão por 24 horas à $25 \pm 2^{\circ} \mathrm{C}, 70 \pm 10 \%$ de umidade relativa e fotofase de 14 horas. A duração do ciclo (ovo-adulto); a porcentagem de parasitismo e de emergência; o número de parasitoides emergidos; a longevidade de machos e de fêmeas, a razão sexual ( $\mathrm{RS}=$ número de fêmeas/ número de adultos) e a largura da cápsula cefálica de machos e de fêmeas foram avaliadas. O sexo dos parasitoides foi determinado pelas características morfológicas da antena e abdome (PARON 1999).

O experimento foi conduzido em esquema fatorial $4 \times 4$ (idades do parasitoide e do hospedeiro) em delineamento inteiramente casualizado, com três repetições, constituídas por um grupo de quatro pupas de A. gemmatalis e 10 e 20 repetições constituídas por 10 machos e 20 fêmeas, escolhidos(as) nos descendentes de cada tratamento para longevidade e largura da cápsula cefálica de machos e de fêmeas, respectivamente. Os dados foram submetidos à análise de variância (ANOVA) e os modelos escolhidos baseados na significância dos coeficientes de regressão utilizando o teste "t" de Student adotando o nível de $5 \%$ de probabilidade, no coeficiente de determinação e no fenômeno biológico estudado.

\section{RESULTADOS}

Na proporção utilizada nesse estudo, o parasitismo por $T$. diatraeae em pupas de A. gemmatalis foi $100 \%$ em todas as combinações de idades (parasitoides x hospedeiros).

A porcentagem de emergência de $T$. diatraeae não apresentou interação entre as idades do parasitoide e hospedeiro $(\mathrm{F}=1,6230$; $\mathrm{p}=0,1505)$ e entre idade do hospedeiro $(\mathrm{F}=2,6670 ; \mathrm{p}=0,0643)$ (Tabela 1), mas isoladamente o aumento da idade do parasitoide reduziu esse parâmetro $(\mathrm{F}=7,3040 ; \mathrm{p}=0,0007)$ com os dados ajustando-se à uma equação linear (Tabela 3).

Tabela 1. Emergência (\%), número de descendentes, duração do ciclo (dias) e razão sexual (número) de Trichospilus diatraeae Cherian \& Margabandhu (Hymenoptera: Eulophidae) com quatro idades, emergidos de pupas de Anticarsia gemmatalis (Hübner) (Lepidoptera: Noctuidae) com quatro períodos de desenvolvimento. $25 \pm 2^{\circ} \mathrm{C}, 70 \pm 10 \%$ de umidade relativa e 14 horas de fotofase.

\begin{tabular}{|c|c|c|c|c|}
\hline \multirow{2}{*}{$\begin{array}{c}\text { Idade de } \\
\text { T. diatraeae (h) }\end{array}$} & \multicolumn{4}{|c|}{ Idade de pupas de $A$. gemmatalis (h) } \\
\hline & 0-24 & $24-48$ & 48-72 & $72-96$ \\
\hline & \multicolumn{4}{|c|}{ Emergência $(\% \pm \text { erro padrão) })^{\text {ns }}$} \\
\hline $0-24$ & $75,00 \pm 0,00$ & $83,33 \pm 8,33$ & $58,33 \pm 8,33$ & $58,33 \pm 8,33$ \\
\hline $24-48$ & $100,0 \pm 0,00$ & $75,00 \pm 14,43$ & $91,67 \pm 8,33$ & $83,33 \pm 8,33$ \\
\hline $48-72$ & $100,0 \pm 0,00$ & $83,33 \pm 16,67$ & $100,00 \pm 0,00$ & $50,00 \pm 14,43$ \\
\hline \multirow[t]{2}{*}{$72-96$} & $91,67 \pm 8,33$ & $75,00 \pm 14,43$ & $91,67 \pm 8,33$ & $50,00 \pm 14,43$ \\
\hline & \multicolumn{4}{|c|}{ 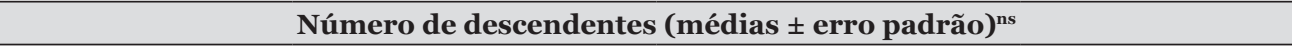 } \\
\hline $0-24$ & $175,00 \pm 20,52$ & $214,08 \pm 20,79$ & $113,92 \pm 18,32$ & $97,75 \pm 30,01$ \\
\hline $24-48$ & $211,83 \pm 35,96$ & $194,75 \pm 35,49$ & $171,17 \pm 18,72$ & $133,08 \pm 42,53$ \\
\hline $48-72$ & $182,75 \pm 17,01$ & $142,83 \pm 40,18$ & $178,42 \pm 08,48$ & $124,83 \pm 33,63$ \\
\hline \multirow[t]{2}{*}{$72-96$} & $197,70 \pm 20,14$ & $195,58 \pm 53,16$ & $189,50 \pm 23,62$ & $130,92 \pm 35,39$ \\
\hline & \multicolumn{4}{|c|}{ Duração do ciclo (dias \pm erro padrão) ${ }^{\text {ns }}$} \\
\hline $0-24$ & $16,44 \pm 0,40$ & $17,92 \pm 0,43$ & $17,17 \pm 0,63$ & $16,11 \pm 0,59$ \\
\hline $24-48$ & $16,50 \pm 0,14$ & $17,72 \pm 0,15$ & $17,64 \pm 0,70$ & $16,44 \pm 0,80$ \\
\hline $48-72$ & $16,67 \pm 0,22$ & $19,17 \pm 0,17$ & $16,75 \pm 0,25$ & $16,56 \pm 0,29$ \\
\hline \multirow[t]{2}{*}{$72-96$} & $16,81 \pm 0,10$ & $17,69 \pm 0,19$ & $16,75 \pm 0,38$ & $16,50 \pm 0,50$ \\
\hline & \multicolumn{4}{|c|}{ Razão sexual (médias \pm erro padrão) ${ }^{\text {ns }}$} \\
\hline $0-24$ & $0,97 \pm 0,00$ & $0,97 \pm 0,00$ & $0,96 \pm 0,00$ & $0,95 \pm 0,01$ \\
\hline $24-48$ & $0,96 \pm 0,01$ & $0,97 \pm 0,00$ & $0,96 \pm 0,00$ & $0,95 \pm 0,01$ \\
\hline $48-72$ & $0,97 \pm 0,00$ & $0,99 \pm 0,00$ & $0,96 \pm 0,00$ & $0,96 \pm 0,02$ \\
\hline $72-96$ & $0,98 \pm 0,00$ & $0,99 \pm 0,00$ & $0,97 \pm 0,00$ & $0,95 \pm 0,03$ \\
\hline
\end{tabular}

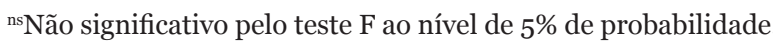


A largura da cápsula cefálica de machos, efeito idade do parasitoide $(\mathrm{F}=0,5710 ; \mathrm{p}=0,6348)$, idade do hospedeiro $(\mathrm{F}=$ $0,3440 ; p=0,7938)$ e interação $(\mathrm{F}=0,2100 ; \mathrm{p}=0,9926)$ e de fêmeas, efeito idade do parasitoide $(\mathrm{F}=0,0930 ; \mathrm{p}=0,9637)$, idade do hospedeiro $(\mathrm{F}=0,5270 ; \mathrm{p}=0,6642)$ e interação $(\mathrm{F}=$ 0,$3210 ; p=0,9678$ ) foram semelhantes entre tratamentos, com variações de 0,52 à $0,57 \mathrm{~mm}$ para machos e de 0,69 à $0,74 \mathrm{~mm}$ para fêmeas (Tabela 2).

O número de descendentes de $T$. diatraeae não foi afetado pela idade de pupas de $A$. gemmatalis $(\mathrm{F}=0,8880 ; \mathrm{p}=0,4614)$ e pela interação (idade de pupas $\mathrm{x}$ idade de parasitoides) $(\mathrm{F}=0,6353$; $p=0,7584$ ) (Tabela 1), mas houve efeito em função da idade dos parasitoides $(\mathrm{F}=4,3761 ; \mathrm{p}=0,0108)$ com os dados ajustando-se à uma equação linear (Tabela 3).

A duração do ciclo de vida de T. diatraeae variou de 16,11 à 19,17 dias e foi semelhante quando analisada na interação entre as idades (parasitoides $\mathrm{x}$ hospedeiros) $(\mathrm{F}=0,9300 ; \mathrm{p}=0,5116) \mathrm{e}$ na idade do hospedeiro $A$. gemmatalis $(\mathrm{F}=0,5300 ; \mathrm{p}=0,6679)$ (Tabela 1), porém significativa quando analisada em função da idade dos parasitoides $(\mathrm{F}=10,6200 ; \mathrm{p}=0,0000)$ ajustando-se a uma função quadrática (Tabela 3).

A interação entre idades (parasitoide $\mathrm{x}$ hospedeiro) $(\mathrm{F}=0,4000$; $\mathrm{p}=0$,9143) e idade do hospedeiro $(\mathrm{F}=0,8000 ; \mathrm{p}=0,4980)$ não afetou a razão sexual de $T$. diatraeae (Tabela 1), mas houve efeito significativo da idade do parasitoide $(\mathrm{F}=4,7000 ; \mathrm{p}=0,0080) \mathrm{com}$ ajuste a uma equação linear (Tabela 3), indicando aparecimento de machos com o avanço da idade das fêmeas de T. diatraeae.

A longevidade de machos de $T$. diatraeae provenientes de quatro idades de fêmeas do parasitoide $(\mathrm{F}=1,5925 ; \mathrm{p}=0,1938)$ e do hospedeiro $(\mathrm{F}=0,6338 ; \mathrm{p}=0,5943)$ foram semelhantes isoladamente ou na interação ( $\mathrm{F}=0,0929 ; \mathrm{p}=0,9997)$, variando de 7,90 a 12,10 dias (Tabela 2). A longevidade de fêmeas foi semelhante quando isolado o efeito da idade do hospedeiro, $A$. gemmatalis $(\mathrm{F}=2,3240 ; \mathrm{p}=0,0750)$ e na interação $(\mathrm{F}=1,2370$; $\mathrm{p}=0,2714$ ) (Tabela 2), porém houve efeito quando se avaliou a idade do parasitoide isoladamente $(\mathrm{F}=4,8500 ; \mathrm{p}=0,0026) \mathrm{com}$ redução linear na longevidade de fêmeas (Tabela 3 ).

\section{DISCUSSÃO}

O parasitismo semelhante entre tratamentos mostra que T. diatraeae não reconhece hospedeiros mais velhos assim como relatado para Meteorus gyrator (Thun.) (Hymenoptera: Braconidae) e Diadegma mollipla (Holmgren) (Hymenoptera: Ichneumonidae) (BELL \& WEAVER 2008; NOFEMELA \& KFIR 2008) e, também, não detecta características físicas e químicas do hospedeiro como relatado para Trichogramma pretiosum Riley e para Trichogramma acacioi Brun, Moraes \& Soares (Hymenoptera: Trichogrammatidae) em ovos de Anagasta kuehniella (Zeller) (Lepidoptera: Pyralidae) (Pratissoli et al. 2005).

Tabela 2. Longevidade e largura da cápsula cefálica de machos e de fêmeas de Trichospilus diatraeae Cherian \& Margabandhu (Hymenoptera: Eulophidae) com quatro idades, emergidos de pupas de Anticarsia gemmatalis (Hübner) (Lepidoptera: Noctuidae) com quatro períodos de desenvolvimento. $25 \pm 2^{\circ} \mathrm{C}, 7 \mathrm{O} \pm 10 \%$ de umidade relativa e 14 horas de fotofase.

\begin{tabular}{|c|c|c|c|c|}
\hline \multirow{2}{*}{$\begin{array}{c}\text { Idade de } \\
\text { T. diatraeae (h) }\end{array}$} & \multicolumn{4}{|c|}{ Idade de pupas de $A$. gemmatalis (h) } \\
\hline & $0-24$ & 24-48 & 48-72 & 72-96 \\
\hline & \multicolumn{4}{|c|}{ 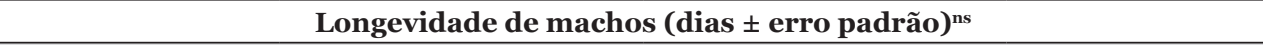 } \\
\hline $0-24$ & $10,40 \pm 1,56$ & $11,40 \pm 1,40$ & $9,70 \pm 1,81$ & $7,90 \pm 2,11$ \\
\hline $24-48$ & $11,40 \pm 1,88$ & $12,10 \pm 1,81$ & $11,00 \pm 1,50$ & $9,00 \pm 2,33$ \\
\hline $48-72$ & $10,00 \pm 1,22$ & $9,50 \pm 1,29$ & $9,80 \pm 1,10$ & $8,00 \pm 1,07$ \\
\hline \multirow[t]{2}{*}{$72-96$} & $10,00 \pm 1,96$ & $10,40 \pm 2,44$ & $9,50 \pm 1,50$ & $8,90 \pm 0,50$ \\
\hline & \multicolumn{4}{|c|}{ 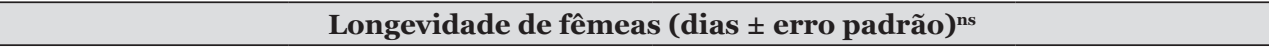 } \\
\hline $0-24$ & $14,95 \pm 0,96$ & $14,75 \pm 1,05$ & $13,00 \pm 0,94$ & $10,90 \pm 1,44$ \\
\hline $24-48$ & $13,85 \pm 1,10$ & $13,70 \pm 0,99$ & $13,80 \pm 0,99$ & $9,50 \pm 1,09$ \\
\hline $48-72$ & $11,65 \pm 1,04$ & $11,75 \pm 1,15$ & $12,75 \pm 1,22$ & $12,05 \pm 0,93$ \\
\hline \multirow[t]{2}{*}{$72-96$} & $11,65 \pm 0,75$ & $11,80 \pm 0,79$ & $12,45 \pm 0,90$ & $10,55 \pm 0,86$ \\
\hline & \multicolumn{4}{|c|}{ Cápsula cefálica de machos $(\mathrm{mm})\left(\right.$ médias \pm erro padrão) ${ }^{\mathrm{ns}}$} \\
\hline $0-24$ & $0,54 \pm 0,02$ & $0,53 \pm 0,03$ & $0,54 \pm 0,02$ & $0,54 \pm 0,02$ \\
\hline $24-48$ & $0,52 \pm 0,02$ & $0,57 \pm 0,02$ & $0,56 \pm 0,02$ & $0,54 \pm 0,03$ \\
\hline $48-72$ & $0,54 \pm 0,03$ & $0,55 \pm 0,02$ & $0,55 \pm 0,03$ & $0,53 \pm 0,02$ \\
\hline \multirow[t]{2}{*}{$72-96$} & $0,52 \pm 0,03$ & $0,55 \pm 0,02$ & $0,53 \pm 0,02$ & $0,53 \pm 0,03$ \\
\hline & \multicolumn{4}{|c|}{ Cápsula cefálica de fêmeas $(\mathrm{mm})$ (médias \pm erro padrão) ${ }^{\mathrm{ns}}$} \\
\hline $0-24$ & $0,72 \pm 0,04$ & $0,74 \pm 0,04$ & $0,74 \pm 0,04$ & $0,74 \pm 0,03$ \\
\hline $24-48$ & $0,73 \pm 0,04$ & $0,69 \pm 0,04$ & $0,74 \pm 0,04$ & $0,73 \pm 0,03$ \\
\hline $48-72$ & $0,72 \pm 0,03$ & $0,73 \pm 0,03$ & $0,69 \pm 0,03$ & $0,70 \pm 0,03$ \\
\hline $72-96$ & $0,71 \pm 0,03$ & $0,69 \pm 0,03$ & $0,73 \pm 0,04$ & $0,69 \pm 0,03$ \\
\hline
\end{tabular}

${ }_{\text {ns }}$ ão significativo pelo teste $\mathrm{F}$ ao nível de $5 \%$ de probabilidade.

Tabela 3. Modelos matemáticos usados para descrever o efeito de quatro idades (o-24; 24-48; 48-72 e 72-96 horas) de fêmeas de Trichospilus diatraeae Cherian \& Margabandhu (Hymenoptera: Eulophidae) em pupas do hospedeiro alternativo Anticarsia gemmatalis (Hübner) (Lepidoptera: Noctuidae) com até 96 horas de idade. $25 \pm 2^{\circ} \mathrm{C}, 70 \pm 10 \%$ de umidade relativa e 14 horas de fotofase.

\begin{tabular}{lcccc}
\hline \multicolumn{1}{c}{ Parâmetro Biológico } & Modelo Matemático & $\mathbf{R}^{2}$ & F & P \\
\hline Emergência (\%) & $\hat{\mathrm{y}}=101,0421-8,7502^{*} \mathrm{x}$ & 0,3445 & 7,3591 & 0,0168 \\
Número de descendentes & $\hat{\mathrm{y}}=224,4049-23,4090^{*} \mathrm{x}$ & 0,5444 & 16,7307 & 0,0011 \\
Duração do ciclo (dias) & $\hat{\mathrm{y}}=14,7270+2,5750 \mathrm{0}-0,5480^{*} \mathrm{x}^{2}$ & 0,5757 & 8,8191 & 0,0038 \\
Razão sexual & $\hat{\mathrm{y}}=0,9810-0,0064^{*} \mathrm{x}$ & 0,3920 & 9,0253 & 0,0095 \\
Longevidade de fêmeas (dias) & $\hat{\mathrm{y}}=14,1500-0,0284^{*} \mathrm{x}$ & 0,2713 & 5,2111 & 0,0386 \\
\hline
\end{tabular}

Longevidade de fêmeas (dias) 
A redução na emergência de descendentes de $T$. diatraeae com o aumento da idade de suas fêmeas pode ser atribuída à diminuição da capacidade de parasitismo e do número de ovos em função do envelhecimento (UÇKAN \& GÜLLEL 2002; JACAS et al. 2005) ou ao aumento da mortalidade dos parasitoides em estágios avançados dos hospedeiros, comparado a estágios mais jovens. Além disso, em alguns casos, hospedeiros mortos podem conter larvas do parasitoide sugerindo que não houve tempo do parasitoide completar o ciclo (MONNERAT et al. 2002; WANG \& LIU 2002; Amalin et al. 2005; Henry et al. 2005; Nofemela \& KFIR 2008).

Fêmeas e machos de $T$. diatraeae em pupas de A. gemmatalis apresentaram largura da cápsula cefálica semelhante ao observado quando criados em pupas de D. sacharalis (GRANCE 2010) e à outras espécies do gênero Trichospilus (Ubaidillah 2006) com longevidade satisfatória para sua utilização em programas de controle biológico (FÁvero 2009; GrANCE 2010). O período de sobrevivência da progênie é importante para permitir que o parasitoide encontre seus hospedeiros (ROITBERg et al. 2001; Harvey et al. 2004) e, no ambiente, sua escassez pode estimular o parasitoide a continuar procurando hospedeiros por maior tempo, controlando a ovulação ou reabsorvendo ovos e aumentando sua longevidade (Silva-Torres et al. 2009). Fêmeas de Trichogramma platneri Nagarkatti (Hymenoptera: Trichogrammatidae) alocam mais recursos para a sobrevivência visando aumentar o tempo de busca em situações de escassez do hospedeiro (HoHMann \& LucK 2004).

A obtenção de descendentes de T. diatraeae com até 96 horas de idade desse parasitoide em pupas do hospedeiro alternativo $A$. gemmatalis com até essa idade, em tubos de vidro e sem chance de escolha, indica que $T$. diatraeae pode utilizar os recursos desse hospedeiro corroborando Paron \& Berti Filho (2000). Isso concorda com o relatado para Melittobia acasta (Walker) (Hymenoptera: Eulophidae) (IMANDEH 2006) e é importante para a criação massal, pois esse parasitoide pode ser usado com até 96 horas de idade sem reduzir suas características reprodutivas. Assim, pupas do hospedeiro podem ser coletadas em períodos programados com até 96 horas de intervalo, otimizando a mão-de-obra sem interferir na biologia de $T$. diatraeae. Uma característica importante para um hospedeiro alternativo é não intervir na biologia de parasitoides (KIVAN \& KILIC 2004; CHONG \& Oetting 2007; FÁvero 2009; Pereira et al. 2009b).

O número de indivíduos emergidos de T. diatraeae não se alterou após a escassez de hospedeiros em resposta à imprevisibilidade de encontros com hospedeiros viáveis como relatado para Oomyzus sokolowskii Kurdjumov (Hymenoptera: Eulophidae) e Anagyrus kamali Moursi (Hymenoptera: Encyrtidae) em larvas de Plutella xylostella (L.) (Lepidoptera: Plutellidae) e larvas e adultos de Maconellicoccus hirsutus Green (Hemiptera: Pseudococcidae), respectivamente (SAgarra \& Vincent 1999; Silva-Torres et al. 2009). Além disso, a densidade dos parasitoides foi suficiente para ovipositar um número de ovos que compensasse a baixa qualidade do hospedeiro, como relatado para Anagyrus sp. nov. nr. sinope Noyes \& Menezes (Hymenoptera: Encyrtidae) em Phenacoccus madeirensis Green (Hemiptera: Pseudococcidae) (Chong \& Oetting 2007). O hospedeiro pode suportar número limitado de descendentes (CoOpERBAND et al. 2003) e esse limite foi alcançado em função da densidade de parasitoides que reduziram a capacidade defensiva de $A$. gemmatalis (ANDRADE $e t$ al. 2010).

Independentemente da idade das pupas de A. gemmatalis, a duração do ciclo de vida de $T$. diatraeae não foi afetada. Isto indica que pupas desse hospedeiro podem não apresentar resposta imune diferencial contra imaturos de parasitoides, pois esse mecanismo de defesa (taxa de encapsulação e produção de toxinas) tem alto custo metabólico (Schmidt et al. 2001; SchmidHempel 2005). Esse resultado difere do que foi observado em pupas de Bombyx mori L. (Lepidoptera: Bombycidae) com 24 horas, quando parasitadas por Palmistichus elaeisis Delvare \& LaSalle (Hymenoptera: Eulophidae) pois houve aumento na duração do ciclo de vida desse parasitoide (PEREIRA et al. 2009a).

A variabilidade da razão sexual de $T$. diatraeae com a idade de suas fêmeas ocorreu, pois progênie e razão sexual de parasitoides variam com o hospedeiro e idade do parasitoide e hospedeiro (UÇKAN \& GÜllel 2002; Nofemela \& KFIR 2008; PASTORI et al. 2010), com o tamanho do hospedeiro (Zaviezo \& Mills 2000) e com o ajuste que parasitoides podem fazem na razão sexual de seus descendentes e no número de ovos depositados em seus hospedeiros (GoDFray 1994). Além disso, descendentes do parasitoide podem ajustar a absorção de nutrientes com a disponibilidade dos recursos do hospedeiro (HäCKERMANn et al. 2007), mas os mecanismos de ajuste utilizados por fêmeas e imaturos de T. diatraeae ainda não foram desvendados.

Pupas do hospedeiro alternativo A. gemmatalis com até 96 horas não afetaram o desenvolvimento de $T$. diatraeae, mas isso pode diferir entre espécies e/ ou populações de parasitoides e hospedeiros devido aos métodos experimentais ou biologia das espécies (WANG et al. 1999). As fêmeas de T. diatraeae com até 24 horas já estão aptas à parasitar, mas o aumento da idade dessas fêmeas altera a emergência, duração do ciclo, número de descendentes, razão sexual e longevidade de fêmeas. A idade de maior atividade e fecundidade de parasitoides é importante para se decidir o momento adequado para estudos de comportamento (KIVAN \& KILIC 2004; Silva-Torres et al. 2009) e otimizar a previsão de liberação em campo visando obter níveis significativos de parasitismo (SAGARRA \& VINCENT 1999). Por exemplo, Ceratogramma etiennei Delvare (Hymenoptera: Trichogrammatidae) deve ser utilizado com um a dois dias após a emergência (Amalin et al. 2005) e P. elaeisis com três a quatro dias (Pereira et al. 2009a). No entanto, fêmeas de T. diatraeae reduzem sua fecundidade e reprodução com o avanço de sua idade.

Trichospilus diatraeae com até 96 horas de idade se reproduz satisfatoriamente em pupas do hospedeiro alternativo $A$. gemmatalis com idade de até 96 horas, embora o aumento da idade das fêmeas de $T$. diatraeae promova redução de alguns parâmetros.

\section{AGRADECIMENTOS}

Ao Conselho Nacional de Desenvolvimento Científico e Tecnológico (CNPq), à Coordenação de Aperfeiçoamento de Pessoal de Nível Superior (CAPES) e à Fundação de Amparo à Pesquisa do Estado de Minas Gerais (FAPEMIG) pelo suporte financeiro.

\section{REFERÊNCIAS}

Amalin, D.M., J.E. Peña \& R.E. Duncan, 2005. Effects of host age, female parasitoid age, and host plant on parasitism of Ceratogrammaetiennei(Hymenoptera:Trichogrammatidae). Florida Entomologist, 88: 77-82.

Andrade, G.S., J.E. Serrão, J.C. Zanuncio, T.V. Zanuncio, G.L.D. Leite \& R.A. Polanczy, 2010. Immunity of an alternative host can be overcome by higher densities of its parasitoids Palmistichus elaeisis and Trichospilus diatraeae. PLoS ONE, 5: e13231.

Bell, H.A. \& R.J. Weaver, 2008. Ability to host regulate determines host choice and reproductive success in the gregarious ectoparasitoid Eulophus pennicornis (Hymenoptera: Eulophidae). Physiological Entomology, 33: 62-72.

Bouček, Z., 1976. The African and Asiatic species of Trichospilus and Cotterellia (Hymenoptera, Eulophidae). Bulletin of Entomological Research, 65: 669-681.

Chong, J.H. \& R.D. Oetting, 2007. Functional response and progeny production of the Madeira mealybug parasitoid, 
Anagyrus sp. nov. nr. sinope: The effect of host stage preference. Biological Control, 41: 78-85.

Cooperband, M.F., R.W. Matthews \& S.B. Vinson, 2003. Factors affecting the reproductive biology of Melittobia digitata and failure to meet the sex ratio predictions of Hamilton's local mate competition theory. Entomologia Experimentalis et Applicata, 109: 1-12.

Fávero, K., 2009. Biologia e técnicas de criação de Trichospilus diatreaea (Hymenoptera: Eulophidae) em pupas de Tenebrio molitor (Coleoptera: Tenebrionidae) e Diatraea saccharalis (Lepidoptera: Crambidae). Dissertação de Mestrado. Universidade Federal da Grande Dourados, Dourados, 63p.

Godfray, H.C.J., 1994. Parasitoids: Behavioral and evolutionary ecology. Princeton, Princeton University Press, 488p.

Grance, E.L.V., 2010. Potencial de Trichospilus diatraeae (Hymenoptera: Eulophidae) para o controle de Diatraeae saccharalis (Lepidoptera: Crambidae) em cana-de-açúcar. Dissertação de Mestrado. Universidade Federal da Grande Dourados, Dourados, 53p.

Greene, G.L., N. C. Leppla \& W.A. Dickerson, 1976. Velvetbean caterpillar: A rearing procedure and artificial diet. Journal of Economic Entomology, 69: 487-488.

Häckermann, J., A.S. Rott, \& S. Dorn, 2007. How two different host species influence the performance of a gregarious parasitoid: Host size is not equal to host quality. Journal of Animal Ecology,76: 376-383.

Harvey, J.A. \& M.R. Strand, 2002. The developmental strategies of endoparasitoid wasps vary with host feeding ecology. Ecology, 83: 2439-2451.

Harvey, J.A., T.M. Bezemer, J.A. Elzinga \& M.R. Strand, 2004 Development of the endoparasitoid Microplitis demolitor: Host quality does not increase with host age and size. Ecological Entomology, 29: 35-43.

Henry, L.M., D.R. Gillespie \& B.D. Roitberg, 2005. Does mother really know best? Oviposition preference reduces reproductive performance in the generalist parasitoid Aphidius ervi. Entomologia Experimentalis et Applicata, 116: 167-174.

Hohmann, C.L. \& R. Luck, 2004. Effect of host availability and egg load in Trichogramma platneri Nagarkatti (Hymenoptera: Trichogrammatidae) and its consequences on progeny quality. Brazilian Archives of Biology and Technology, 47: 413-422.

Imandeh, N.G., 2006. Effect of the pupal age of Calliphora erythrocephala (Diptera: Calliphoridae) on the reproductive biology of Melittobia acasta (Walker) (Hymenoptera: Chalcidoidea: Eulophidae). Entomological Science, 9: 7-11.

Jacas, J.A., J.E. Peña \& R.E. Duncan, 2005. Successful oviposition and reproductive biology of Aprostocetus vaquitarum (Hymenoptera: Eulophidae): A predator of Diaprepes abbreviatus (Coleoptera: Curculionidae). Biological Control, 33: 352-359.

Kivan, M. \& N. Kilic, 2004. Influence of host species and age on host preference of Trissolcus semistriatus. Biological Control, 49: $553-562$.

Lin, L.A. \& A.R. Ives, 2003. The effect of parasitoid host-size preference on host population growth rates: an example of Aphidius colemani and Aphis glycines. Ecological Entomology, 28: 542-550.

Monnerat, R.G., A.A. Kirk \& E.D. Bordat, 2002. Biology of Diadegma sp. (Hymenoptera: Ichneumonidae), a parasitoid of Plutella xylostella (L.) (Lepidoptera: Yponomeutidae), from Reunion Island. Neotropical Entomology, 31: 271-274.

Nofemela, R.S. \& R. Kfir, 2008. Diadegma mollipla parasitizing Plutella xylostella: host instar preference and suitability. Entomologia Experimentalis et Applicata,126: 9-17.

Paron, M.R., 1999. Bioecologia de Trichospilus diatraeae Cherian \& Magabandhu, 1942 (Hymenoptera: Eulophidae), endoparasitoide de pupas de Lepidoptera. Tese de Doutorado, ESALQ/USP, Piracicaba, 57p.

Paron, M.R. \& E. Berti Filho, 2000. Capacidade reprodutiva de Trichospilus diatraeae (Hymenoptera: Eulophidae) em pupas de diferentes hospedeiros (Lepidoptera). Scientia Agricola, 57: 355-358.

Pastori, P.L., L.B. Monteiro, M. Botton \& D. Pratissoli, 2010. Efeito da idade do parasitoide e do hospedeiro na reprodução de Trichogramma pretiosum Riley (Hymenoptera: Trichogrammatidae) em ovos de Bonagota salubricola (Meyrick) (Lepidoptera: Tortricidae). Arquivos do Instituto Biológico, 77: 349-353.

Pereira, F.F., J.C. Zanuncio, M.T. Tavares, P.L. Pastori, G.C. Jacques \& E.F. Vilela, 2008. New record of Trichospilus diatraeae as a parasitoid of the eucalypt defoliator Thyrinteina arnobia in Brazil. Phytoparasitica, 36: 304-306.

Pereira, F.F., J.C. Zanuncio, J.E. Serrão, H.N. Oliveira, K. Fávero \& E.L.V. Grance ELV, 2009. Progênie de Palmistichus elaeisis Delvare \& LaSalle (Hymenoptera: Eulophidae) parasitando pupas de Bombyx mori L. (Lepidoptera: Bombycidae) de diferentes idades. Neotropical Entomology, 38: 660-664.

Pereira, F.F., J.C. Zanuncio, J.E. Serrão, P.L. Pastori \& F.S. Ramalho, 2009b. Reproductive performance of Palmistichus elaeisis Delvare and LaSalle (Hymenoptera: Eulophidae) with previously refrigerated pupae of Bombyx mori L. (Lepidoptera: Bombycidae). Brazilian Journal of Biology, 69: 865-869.

Pratissoli, D., J.C. Zanuncio, U.R. Vianna, J.S. Andrade, L.C.M. Zanotti \& A.F. Silva, 2005. Biological characteristics of Trichogramma pretiosum and Trichogramma acacioi (Hym.: Trichogrammatidae), parasitoids of the avocado defoliator Nipteria panacea (Lep.: Geometridae), on eggs of Anagasta kuehniella (Lep.: Pyralidae). Brazilian Archives of Biology and Technology, 48: 7-13.

Rodrigues, M.A.T., 2010. Exigências térmicas e hídricas de Trichospilus diatraeae (Hymenoptera: Eulophidae) em pupas de Diatraea saccharalis (Lepidoptera: Crambidae). Dissertação de Mestrado. Universidade Federal da Grande Dourados, Dourados, 52p.

Roitberg, B.D., G. Boivin \& L.E.M Vet, 2001. Fitness, parasitoids, and biological control: an opinion. Canadian Entomologist, 133: 429-438.

Sagarra, L.A. \& C. Vincent, 1999. Influence of host stage on oviposition, development, sex ratio, and survival of Anagyrus kamali Moursi (Hymenoptera: Encyrtidae), a parasitoid of the hibiscus mealybug, Maconellicoccus hirsutus Green (Homoptera: Pseudococcidae). Biological Control, 15: 51-56.

Schmid-Hempel, P., 2005. Evolutionary ecology of insect immune defenses. Annual Review of Entomology, 50: 529-551.

Schmidt, O., V. Theopold \& M.R. Strand, 2001. Innate immunity and its evasion and suppression by Hymenoptera endoparasitoid. Bioessays, 234: 344-351.

Silva-Torres, C.S.A., R. Barros \& J.B. Torres, 2009. Efeito da idade, fotoperíodo e disponibilidade de hospedeiro no comportamento de parasitismo de Oomyzus sokolowskii Kurdjumov (Hymenoptera: Eulophidae). Neotropical Entomology, 38: 512-519.

Ubaidillah, R., 2006. Eulophine parasitoids of the genus Trichospilus in Indonesia, with the description of two new species (Hymenoptera: Eulophidae). Entomological Science, 9: 217-222.

Uçkan, F. \& A. Gülel, 2002. Age-related fecundity and sex ratio variation in Apanteles galleriae (Hym., Braconidae) and host effect on fecundity and sex ratio of its hyperparasitoid Dibrachys boarmiae (Hym., Pteromalidae). Journal of Applied Entomology, 126: 534-537.

Zaché, B., C.F. Wilcken, R.R. Dacosta \& E.P. Soliman, 2010. Trichospilus diatraeae Cherian \& Margabandhu, 1942 (Hymenoptera: Eulophidae), a new parasitoidof Melanolophia consimilaria (Lepidoptera: Geometridae). Phytoparasitica, 38: 355-357.

Zaviezo, T. \& N. Mills, 2000. Factors influencing the evolution of clutch size in a gregarious insect parasitoid. Journal of Animal Ecology, 69: 1047-1057.

Wang, X-G., S-S. Liu, S-J. Guo \& W-C. Lin, 1999. Effects of 
host stages and temperature on population parameters of Oomyzus sokolowskii, a larval-pupal parasitoid of Plutella xylostella. Biological Control, 44: 391-402.

Wang, X-G. \& S-S. Liu, 2002. Effects of host age on the performance of Diadromus collaris, a pupal parasitoid of Plutella xylostella. Biological Control, 47: 293-307.
Recebido em: 26/04/2011

Aceito em: 24/01/2012

Como citar este artigo:

Pastori, P.L., J.C. Zanuncio, R.O. Silva, F.F. Pereira, R. Azambuja \& J.M.M. Pereira, 2012. Reprodução de Trichospilus diatraeae Cherian \& Margabandhu (Hymenoptera: Eulophidae) em Pupas de Anticarsia gemmatalis Hübner (Lepidoptera: Noctuidae) em Relação à Idade do Parasitoide e Hospedeiro. EntomoBrasilis, 5(1): 37-42.

Acessível em: http://www.periodico.ebras.bio.br/ojs/index.php/ebras/article/view/155
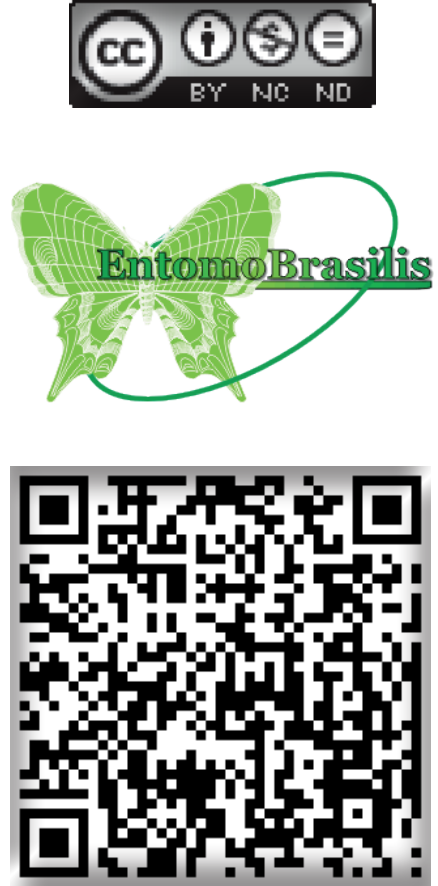